\title{
Director precession and nonlinear waves in nematic liquid crystals under elliptic shear
}

\author{
T. Börzsönyi ${ }^{1, *}$ Á. Buka ${ }^{1}$ A. P. Krekhov ${ }^{2,3}$ O. A. Scaldin ${ }^{3}$ and L. Kramer ${ }^{2}$ \\ ${ }^{1}$ Research Institute for Solid State Physics and Optics, Hungarian Academy of Sciences, H-1525 Budapest, P.O.B.49, Hungary \\ ${ }^{2}$ Physikalisches Institut, Universität Bayreuth, D-95440 Bayreuth, Germany \\ ${ }^{3}$ Institute of Molecule and Crystal Physics, Russian Academy of Sciences, 450025 Ufa, Russia \\ * Present address: Groupe de Physique des Solides, CNRS UMR 75-88, Universités Paris VI et VII, Tour 23, 2 place Jussieu, \\ 75251 Paris Cedex 05, France
}

\begin{abstract}
Elliptic shear applied to a homeotropically oriented nematic above the electric bend Fréedericks transition (FT) generates slow precession of the director. The character of the accompanying nonlinear waves changes from diffusive phase waves to dispersive ones exhibiting spirals and spatiotemporal chaos as the FT is approached from above. An exact solution of the flow alignment equations captures the observed precession and predicts its reversal for non-flow aligning materials. The FT transforms into a Hopf bifurcation opening the way to understand the wave phenomena.
\end{abstract}

Liquid crystals (LCs) exhibit a multitude of interesting nonlinear dynamical phenomena, like electrically and thermally driven convection, flow-induced and optical instabilities [1.22. Nonlinear dispersive waves as observed in particular in excitable and oscillatory chemical reactions [3] are rarely seen in LCs. An exception are the $2 \mathrm{D}$ waves that modulate the oscillating bimodal pattern found in electroconvection of nematics [4] for which only a phenomenological description has been developed.

Actually, nonlinear dispersive waves are obtained in a much simpler situation when a local precession of the director is generated by an elliptic shear flow in a homeotropically anchored, Fréedericks distorted nematic slab. As will be shown here, the dynamics can then be nearly conservative, in contrast to the well studied situation where the director is set into motion by a rotating magnetic or electric field [5] and the dynamic phenomena are limited by the strong dissipation. In the presence of elliptic shear the FT transforms into a Hopf bifurcation and the observed transition to chaos can possibly be related to the Benjamin-Feir instability, see e.g. [6].

Elliptic shear flow in homeotropically aligned nematics has been studied intensively in the past in view of an instability leading to roll patterns [7,8]. For that purpose rather large shear angles are needed and no external field is applied. The only previous study of the Fréedericks distorted state in the presence of elliptic shear was, to our knowledge, carried out by Dreyfus and Pieranski. They observed some of the phenomena discussed below without a conclusive interpretation [9].

In this work the nematic layer (thickness $d$ ) has been sandwiched between clean, $\mathrm{SnO}_{2}$ coated float glass plates ( $x y$ plane) resulting in homeotropic anchoring (nematic director $\hat{\mathbf{n}}$ perpendicular to the bounding plates) of the substance Phase 5A (Merck). A voltage $U$ applied across the sample induces at a critical value $U_{F}$ a bend Fréedericks transition (FT) (the dielectric anisotropy $\varepsilon_{a}$ of Phase $5 \mathrm{~A}$ is negative ). Above $U_{F}$ the director tilts away from the $z$ direction. The tilt angle $\theta$ can be controlled by varying $U$. The degeneracy with respect to the azimuthal angle $\phi$ leads to the well known "schlieren texture" seen between crossed polars 10. It contains defects (umbilics) with topological charge \pm 1 depending on whether the in-plane director makes a $2 \pi$ or $-2 \pi$ rotation on a closed loop around them. These can be distinguished by rotating the crossed polars.

Elliptic shear flow has been generated by oscillating the bottom glass plate in the $x$ direction $\left[x(t)=A_{x} \cos (\omega t)\right]$ and the top plate along $y\left[y(t)=A_{y} \cos (\omega t+\Phi)\right.$; unless stated otherwise the phase shift will be $\Phi=-\pi / 2]$. The oscillations were produced by loud speakers. The precession of the director was observed as the local oscillation of the transmitted light intensity that results in wave-like propagation of bright and dark domains. The recordings were studied by digital image analysis. Details of the experimental setup are described in 11, 12].

At sufficiently high voltages the director orientation varies slowly in space and precesses almost homogeneously in time (Fig.1] bottom). Around $1.2 U_{F}$ inhomogeneities emit traveling waves and umbilics generate spiral waves, very similar to those observed in oscillatory and excitable chemical reactions [3]. The longer waves in the background originate from the lateral cell boundaries (Fig. 1 middle). At lower voltages spiral pairs seem to be created spontaneously (without umbilics) and one observes spatio-temporal chaos (Fig.1 top).

In Fig 2 the dependence of the precession frequency $\Omega$ on the voltage is shown for two temperatures. Clearly $\Omega$ behaves differently in the different regimes (see Fig.1). The dependence of $\Omega$ on the frequency and amplitude of the applied shear is given in Fig. 3. The linear dependencies can be understood from symmetry arguments and dimensional analysis as shown in the following.

In order to describe the director precession we look for solutions of the nematodynamic equations where the director $\hat{\mathbf{n}}$ and the velocity $\mathbf{v}=\left(v_{x}, v_{y}, 0\right)$ depend only on 
the coordinate $z$ and time $t$. Measuring lengths in units of $d$, times in units of $1 / \omega$, we introduce

$$
\begin{gathered}
k_{i}=K_{i i} / K_{33}, \lambda=\alpha_{3} / \alpha_{2}, \tau_{d}=\gamma_{1} d^{2} / K_{33}, \gamma_{1}=\alpha_{3}-\alpha_{2} \\
\epsilon=1 /\left(\tau_{d} \omega\right), e^{2}=U^{2} / U_{F}^{2}, U_{F}=\sqrt{K_{33} \pi^{2} /\left(\varepsilon_{0}\left|\varepsilon_{a}\right|\right)}
\end{gathered}
$$

with $K_{i i}$ the elastic constants and $\alpha_{i}$ the Leslie coefficients. Setting $N=n_{x}+i n_{y}, V=v_{x}+i v_{y}$, one can write the nematodynamic equations concisely in complex notation. The torque balance equation [8,10] is

$$
\begin{array}{r}
N_{, t}=\frac{n_{z}}{(1-\lambda)}\left(V_{, z}-\frac{1+\lambda}{2} N\left(N \bar{V}_{, z}+\bar{N} V_{, z}\right)\right) \\
-\epsilon\left\{k_{1} n_{z} n_{z, z z} N-\left[N_{, z z}-N\left(N \bar{N}_{, z z}+\bar{N} N_{, z z}\right) / 2\right]\right. \\
\left.+\left(1-k_{2}\right)\left[N\left(B^{2}+i B_{, z}\right) / 2+i N_{, z} B\right]-\pi^{2} e^{2} n_{z}^{2} N\right\},
\end{array}
$$

with $n_{z}=\sqrt{1-|N|^{2}}, B=i\left(N \bar{N}_{, z}-\bar{N} N_{, z}\right)$, and ${ }_{, i}$ denote derivatives.

For frequencies such that the viscous penetration depth satisfies $l=\sqrt{\gamma_{1} / \rho \omega}>>d[f=\omega /(2 \pi)$ below about $40 \mathrm{kHz}$ for $d=20 \mu \mathrm{m}]$, and small dimensionless shear amplitudes $a=A_{x} / d$ one has a linear flow field, i.e. $V, z=a(\cos t+i b \sin t)$ where $b=A_{y} / A_{x}$ is a measure of ellipticity ( $b=1$ for circular shear).

It is useful to first neglect any space dependence, which is a good approximation when the Ericksen number $a / \epsilon$ [8] is large. Consistently, one then also has to discard the electric field, so that in Eq.(2) one is left with the terms in the first line. Introducing angles by writing $N=\sin \theta \exp i \phi$ one can rewrite the equation as

$$
\begin{aligned}
\theta, t & =a^{\prime}\left(\cos ^{2} \theta-\lambda \sin ^{2} \theta\right)[\cos t \cos \phi+b \sin t \sin \phi], \\
\phi, t & =a^{\prime} \cot \theta[-\cos t \sin \phi+b \sin t \cos \phi]
\end{aligned}
$$

where $a^{\prime}=a /(1-\lambda)$. For rectilinear shear $(b=0)$ one recovers the flow-aligned solution $\cot ^{2} \theta=\lambda, \phi=0$.

Equations (3) represent a conservative, reversible dynamical system. For $\lambda=0$ the director is advected passively by the velocity field and Eqs. (3) separate into

$$
\partial_{t}(\tan \theta \cos \phi)=a \cos t, \partial_{t}(\tan \theta \sin \phi)=a b \sin t .
$$

The solutions for arbitrary initial conditions can easily be written. They describe simple, closed $2 \pi$-periodic orbits, which either include or exclude the origin (homeotropic orientation). Clearly this case can be generalised to arbitrary time dependence of the flow.

For circular flow $(b=1)$ Eqs.(3) are integrable even for $\lambda \neq 0$. Then the terms in square brackets become $\cos (\phi-t)$ and $-\sin (\phi-t)$, respectively. Introducing the phase lag $\varphi=\phi-t$ the equations become autonomous. Transforming them into the 2nd-order ODE

$$
\varphi, t t=\left(\varphi,{ }_{t}+1\right)\left(2 \varphi,{ }_{t}+1\right) \cot \varphi-\lambda a^{\prime 2} \sin \varphi \cos \varphi
$$

one can verify that the quantity

$$
C=\frac{\left(\varphi, t+1-\lambda a^{\prime 2} \sin ^{2} \varphi\right)^{2}}{\left(2 \varphi, t+1-\lambda a^{\prime 2} \sin ^{2} \varphi\right) \sin ^{2} \varphi}
$$

is a constant of motion. Solving for $\varphi, t$ one obtains the (1)period $T$ of the motion as an integral which can be solved analytically giving

$$
T=\int_{0}^{2 \pi} d \varphi / \varphi, t=2 \pi / \sqrt{1-\lambda a^{\prime 2}}
$$

(independent of $C !$ ). For $\lambda \neq 0$ the orbits are in general quasi-periodic. Thus, in addition to the rapid oscillation, the director performs a slow precession with frequency $\Omega=(1-2 \pi / T) \omega \approx(\lambda / 2) a^{\prime 2} \omega$ in physical units. The precession is for flow-aligning materials positive (same sense of rotation as the elliptic shear) and negative otherwise. In Fig. 1 some typical orbits are shown.

Simulations show that for $b \neq 1$ (not too small) and $\lambda \neq 0$ the slow precession persists and has the same sign as for $b=1$. One has $\Omega \approx(\lambda / 2) a^{\prime 2} b \omega$, which can also be derived analytically for small shear flow amplitudes.

The dependence of $\Omega$ on the ellipticity is explored experimentally most easily by keeping $A_{x}=A_{y}$ fixed and changing the phase shift $\Phi$. This driving is actually equivalent to one with phase shift $-\pi / 2$ and $A_{x}^{\prime}=$ $\sqrt{2} A_{x} \cos (\Phi / 2), \quad A_{y}^{\prime}=\sqrt{2} A_{y} \sin (\Phi / 2)$, as can be seen by transforming into a rotated coordinate system. Thus $a^{2} b=\left(A_{x} A_{y} / d^{2}\right)|\sin \Phi|$. In Fig. 2 (insert) the experimental results are compared with the theoretical prediction. $\Omega / \omega$ was always found to be positive as expected for the flow-aligning case. From the magnitude of $\Omega$ at $U / U_{F} \approx 2$ and $T=25 \ldots 30^{\circ} C$ in Figs. 2, 3a, and 3b. we find $\lambda=0.008 \pm 0.004$, which appears consistent with the very small values found in [13] and falls within the experimental uncertainty of those measurements.

Now we discuss the effect of the terms in curly brackets in Eq. (2). These terms represent singular perturbations. They introduce dissipation into the system, which, together with the boundary conditions, produces the attractors. For $b=1, a<<1$ and at lowest order (in $\epsilon$ ), this reduces to a (z-dependent) selection from the family of solutions of the conservative system. Although this approximation breaks down near the bounding plates it can give useful results (see the rectilinear case [14]). One then expects that (aside from the slow precession) the director performs small-amplitude oscillations around the director distribution $\theta_{0}(z)$ obtained from equating the curly brackets in Eq.(2) to zero (with homeotropic boundary conditions $N=0$ ), which corresponds to the usual (static) bend Fréedericks distortion. In this approximation the frequency of the slow precession should be as calculated above. We also analysed slow modulations in the $x y$ plane and found them to give rise to diffusive phase waves as observed well above $U_{F}$.

The experimental results indicate that corrections to the above behavior are important, in particular near 
the FT. We have analysed corrections only in limiting cases and found the contribution to the slow precession to be positive. In particular, one should note that in the presence of circular shear the FT is transformed into a Hopf bifurcation, as can be seen purely from symmetry arguments: below the transition the director rotates symmetrically around the homeotropic orientation. At the FT this symmetry is broken and generically a new frequency develops (no chiral symmetry in the rotating frame) which leads to the precession.

Consequently, near the FT a description in terms of a complex Ginzburg-Landau equation should be possible. For low frequencies $(\epsilon \sim 1)$ and near the $\mathrm{FT}$ we have performed a fairly full analysis. We find the FT to be slightly delayed and modulations in the $x y$ plane to exhibit strong dispersion, which presumably leads to the observed modulational (Benjamin-Feir) instability. For $1>b>b_{c} \approx 0.5$ the behavior remains qualitatively similar. At $b_{c}$ there is a crossover from oscillatory to excitable behavior of the system 15.

In summary: the theory describes the slow precession, at large fields essentially quantitatively. The scenario of the waves on the background of the slow precession (diffusive phase waves at large fields changing to amplitude waves with dispersion and eventually BenjaminFeir chaos at low fields) can be understood qualitatively. There remains to be done a quantitative analysis at low fields as well as an experimental test of the most provocative prediction, namely the reversal of the slow precession for non-flow aligning materials in situations where the elasticity-induced effects are small.

We have observed similar behavior in the nematics MBBA and DOBHOP $\left(\varepsilon_{a}<0\right)$. Also, in 5CB $\left(\varepsilon_{a}>0\right)$ we generated the director precession by inducing the director tilt by surface effects: the bottom plate of the horizontal cell was heated above the phase transition point $\left(34.5^{\circ} \mathrm{C}\right)$ so that a nematic-isotropic interface developed in the $x y$ plane. The surface energy of this interface is minimized when the director encloses an angle $\sim 64^{\circ}$ with the surface normal [16].

We have also performed experiments with linear mechanical vibration along $z$ (compression) generated by piezo crystals $(f \approx 5-100 \mathrm{kHz})$ attached to one of the bounding plates. This presumably induces Poiseuille flow. As before, the slow precession occurred only in the Fréedericks distorted state. Here the phase waves are typically emitted from certain locations in the form of target patterns, which presumably result from spatial inhomogeneities in the flow. The waves behave diffusively (even near $U_{F}$ ), which is probably due to the fact that at the high frequencies used the elastic contributions to the precession are irrelevant [17]. For more details, see [12].

Director precession and phase waves have been observed previously in cells that were excited piezo electrically at frequencies around $50 \mathrm{kHz}[18]$. The piezo crystal formed one of the bounding plates. Phenomena reminis- cent of the phase waves were also seen in planar and homeotropic cells without electric field at frequencies 10 $\mathrm{kHz}<f<1 \mathrm{MHz}$ [19]. There the waves originated from orientational defects at the surface. Although in these cases the precise form of the excitation was not clear we suggest that the mechanism presented here forms the basis of the phenomena.

Finally we note that the precession should be observable also in smectic $\mathrm{C}$ layers excited elliptically. Possibly the effect can be used to measure the analog of $\alpha_{3} / \alpha_{2}$.

We wish to thank P. Coullet, P. Pieranski, T. Tél and M. Khazimullin for illuminating discussions. The hospitality of the Max-Planck-Institute for the Physics of Complex Systems at Dresden, where part of the work was performed, is greatly appreciated. Financial support by OTKA-T014957, EU (TMR ERBFMRXCT960085) DFG (Grant Kr 690/12-1), INTAS (Grant 96-498), and Volkswagen-Stiftung (I/72 920) is gratefully acknowledged.

[1] F. Simoni Nonlinear optical properties of liquid crystals (World Scientific, Singapore, 1997); G. Demeter and L. Kramer Phys. Rev. Lett., in press.

[2] See e.g. Pattern Formation in Liquid Crystals, A. Buka and L. Kramer, editors (Springer-Verlag, New York, 1996).

[3] See e.g. A. de Wit, Adv. Chem. Physics, I. Prigogine and S.A. Rice, editors (John-Wiley, New York, 1999) Vol.109, p. 435 .

[4] M. Sano, H. Kokubo, B. Janiaud, and K. Sato, Progresses in Theoretical Physics, 90, 1 (1993); V. A. Delev, O. A. Scaldin, and A. N. Chuvyrov, Mol. Cryst. Liq. Cryst., 215, 179-186 (1992).

[5] C. Zheng and R.B. Meyer, Phys. Rev. E., 56, 5553 (1997); T. Frisch, S. Rica, P. Coullet, and J. Gilli, Phys. Rev. Lett., 72, 1471 (1994); S. Nasuno and N. Yoshimo and S. Kai, Phys. Rev. E., 51, 1598 (1995).

[6] M. C. Cross and P. C. Hohenberg, Rev. Mod. Phys. 65, 851 (1993).

[7] P. Pieranski and E. Guyon, Phys. Rev. Lett., 39, 1281 (1977); E. Dubois-Violette and F. Rothen, J. Phys. France, 39, 1039 (1978);

[8] E. Dubois-Violette and P. Manneville, in [2].

[9] J.-M. Dreyfus and P. Pieranski. J. Physique, 42, 459 (1981).

[10] P. G. de Gennes and J. Prost, The Physics of Liquid Crystals. Clarendon Press, Oxford, 1993; S. Chandrasekhar, Liquid Crystals. Dover Publication, New York, 1981.

[11] T. Börzsönyi, Á. Buka, A.P. Krekhov, and L. Kramer, Phys. Rev. E., 58, 7419 (1998).

[12] T. Börzsönyi, doctoral dissertation, Budapest, 1998 (in English). 
[13] H. H. Graf, H. Kneppe, and Schneider, Molecular Physics 77, 521 (1992).

[14] A.P. Krekhov, L. Kramer, J. Physique II, 4, 677 (1994), Phys. Rev. E., 53, 4925 (1996).

[15] S.C. Müller, P. Coullet, and D. Walgraef, Chaos, 4, 443 (1994).

[16] S. Faetti, Mol. Cryst. Liq. Cryst., 179, 217 (1990).

[17] In the immediate neighborhood of the FT and at not too high temperatures, a reversal of the precession was observed in Phase 5 and MBBA.

[18] A. N. Chuvyrov, Zh. Eksp. Teor. Fiz., 82, 761 (1982) [Sov. Phys. JETP 55, 451 (1982)]; A. N. Chuvyrov, O. A. Scaldin, and V. A. Delev. Mol. Cryst. Liq. Cryst., 215, 187 (1992).

[19] F. Scudieri, Ann. Phys., 3, 311 (1978).

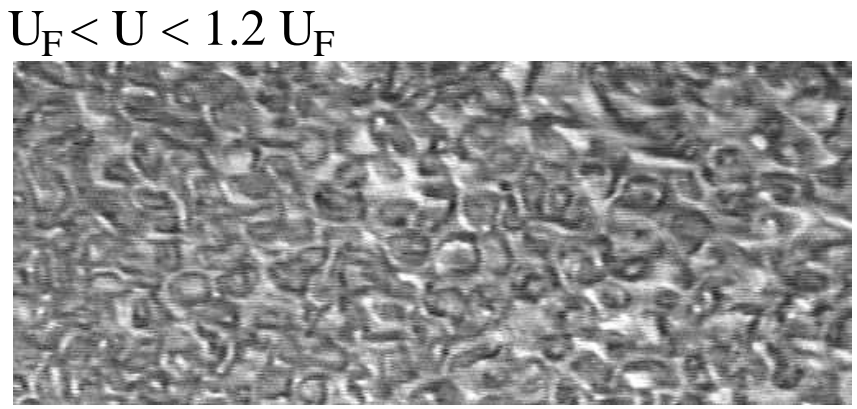

$\mathrm{U} \approx 1.2 \mathrm{U}_{\mathrm{F}}$

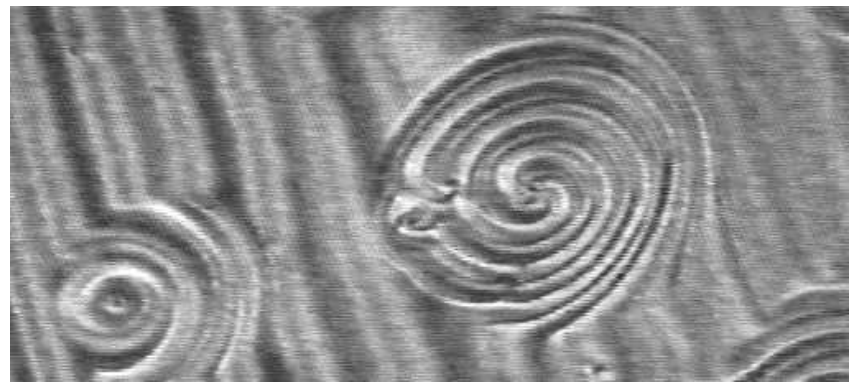

$\mathrm{U} \gtrsim 1.5 \mathrm{U}_{\mathrm{F}}$

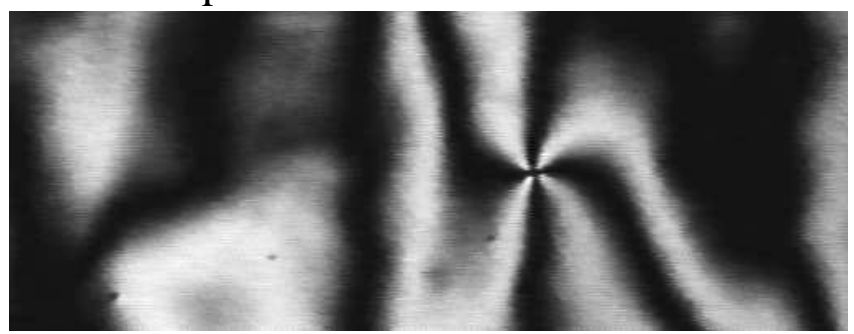

FIG. 1. Snapshots in the different regimes (crossed polars). Circular shear, $A_{x}=A_{y}=3.4 \mu \mathrm{m}, f=155 \mathrm{~Hz}$, and $T=28.2^{\circ} \mathrm{C}$.

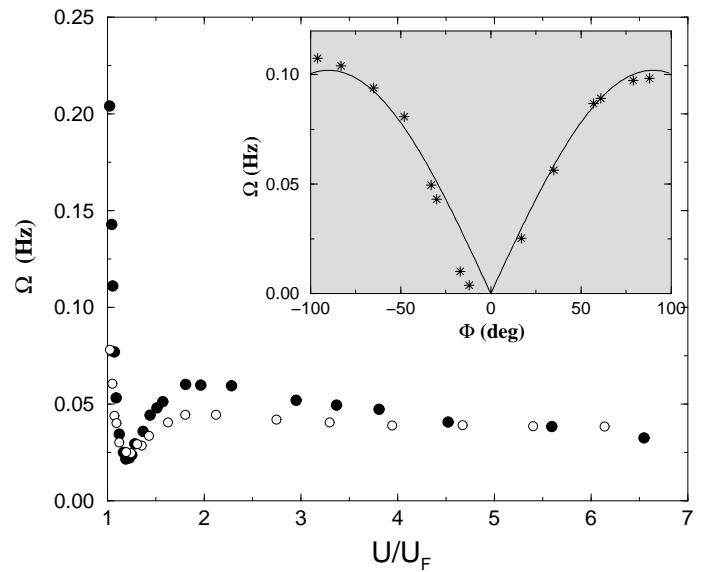

FIG. 2. The precession frequency $\Omega$ as a function of the applied voltage for $T=29^{\circ} \mathrm{C}, U_{F}=8.4 \mathrm{~V}(\bullet)$ and $T=53^{\circ} \mathrm{C}$, $U_{F}=10.7 \mathrm{~V}(\circ)$. The other parameters were: $d=20 \mu \mathrm{m}$, $A_{x}=3.1 \mu \mathrm{m}, A_{y}=3.5 \mu \mathrm{m}, f=155 \mathrm{~Hz}$ and $\Phi=80^{\circ}$. In the insert we show $\Omega$ as a function of the phase shift $\Phi$ for $A_{x}=A_{y}=4 \mu \mathrm{m}, f=122 \mathrm{~Hz}, d=20 \mu \mathrm{m}, U / U_{F}=2.3$ and $T=24.5^{\circ} \mathrm{C}\left(^{*}\right)$. The continuous line shows $\Omega=0.102|\sin \Phi|$.
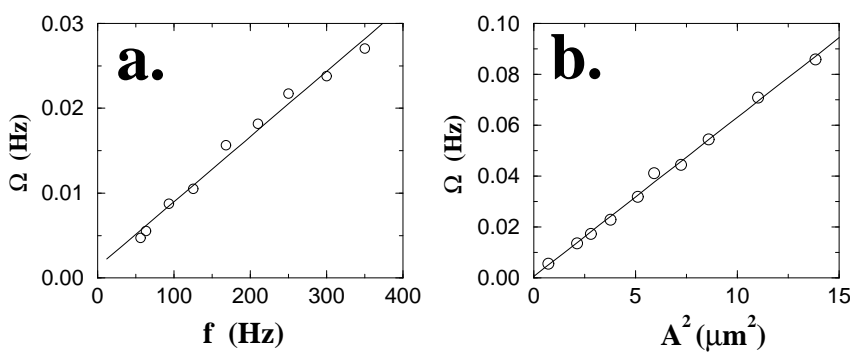

FIG. 3. Precession frequency vs. driving frequency and amplitude. The parameters are: a.) $U / U_{F}=2.3, d=20$ $\mu \mathrm{m}, A_{x}=0.8 \mu \mathrm{m}, A_{y}=1 \mu \mathrm{m}$ and $T=25.5^{\circ} \mathrm{C}$. b. $)$ Circular shear, $d=20 \mu \mathrm{m}, f=122 \mathrm{~Hz}, T=28.5^{\circ} \mathrm{C}$ and $U / U_{F}=2.3$.

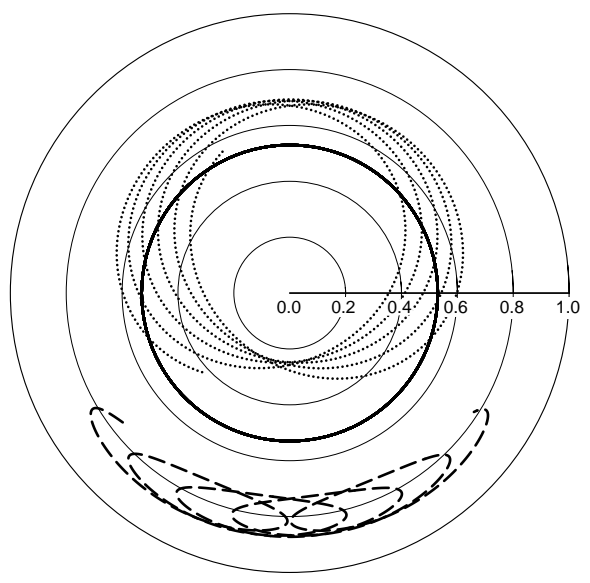

FIG. 4. Three orbits of the in-plane director under circular flow for $\lambda=0.2, a=0.5$. The thick circle represents a pure rapid rotation around the $z$ axis relevant below the FT. (In the elliptic case this orbit transforms into an approximate ellipse.) Other orbits exhibit the slow precession. We show examples with a small average tilt (dotted), expected to be relevant slightly above the $\mathrm{FT}$, and with a large tilt (dashed). 